International Journal of Modern Physics E (C) World Scientific Publishing Company

\title{
HEAVY ION DYNAMICS AND NEUTRON STARS
}

\author{
MASSIMO DI TORO * \\ Laboratori Nazionali del Sud INFN, I-95123 Catania, Italy, \\ and Physics-Astronomy Dept., University of Catania \\ *E-mail:_ditoro@lns.infn.it \\ Received October 07 \\ Revised October 07
}

\begin{abstract}
Some considerations are reported, freely inspired from the presentations and discussions during the Beijing Normal University Meeting on the above Subject, held in July 2007. Of course this cannot be a complete summary but just a collection of personal thougths aroused during the workshop.
\end{abstract}

\section{Introduction}

It is a real pleasure to write a few summary notes on this very stimulating International Workshop held in the nice atmosphere of the Normal University of Beijing. First of all I have to stress the interesting project that was behind the organization of such meeting: try to put together experts working in apparently distant fields of the nuclear research. The Heavy Ion Dynamics studies (in terrestrial laboratories) address typical non-equilibrium properties of nuclear systems. The Compact Stars are objects (not directly probed) which show typical equilibrium properties of a nuclear matter very far away from normal conditions. The link is in fact extremely clear and based on two fundamental topics that have been extensively discussed during the workshop:

- 1. The structure of the effective nuclear interactions in extreme conditions for various degrees of freedom, density, isospin, spin, strangeness....

- 2. The phases of nuclear matter, with particular attention to the high temperature and/or high density phase transitions, chiral vs. deconfinement.

Before passing to a more detailed analysis of the main points discussed at the meeting I would like to make a general comment on the fact that this clear connection between Heavy ion Collisions $(H I C)$ and Neutron Stars $(N S)$ is not much present in the activity of the Radioactive Ion Beam $(R I B)$ Community: the main focus is on nuclear structure problems, studied with "soft" peripheral collisions. More dissipative reactions with exotic beams, testing the Equation of State (EOS) of asymmetric matter, seem not to be of much interest. We are at the level of a 
dogmatic "Doctrina Una Veritas Una" ("One Doctrine One Truth"), not really a scientific approach.

\section{Neutron Stars: A Many-EOS Problem}

The relationship between equilibrium properties of $N S$ (mass/radius, central densities, cooling...) and the EOS of a "Nuclear" matter in various phases (hadronic, mixed, partonic) has emerged very clearly. Unfortunately we have not yet reached the knowledge of an unique EOS which can consistently describe the hadron-quark transition. Very likely such transition is taking place in the interior of some compact stars. In order to make predictions we must refine our knowledge of hadronic and partonic EOS's and suggest some sensitive observables.

\section{Hadronic EOS}

The inclusion of 3-body forces is essential in order to get the correct saturation point for symmetric NM. U.Lombardo has shown that in a non-relativistic BruecknerHartree-Fock $(B H F)$ approach this contribution leads to a more repulsive matter at high baryon and isospin density 1 . The latter point, on a more repulsive isospin potentials (symmetry energy) at high density, appears very important for various aspects:

- We can reconcile a "stiff" EOS for asymmetric matter with the relatively "soft" behavior required in isospin symmetric cases from relativistic heavy ion collisions, in order to account for nucleon/meson production and flows 2 .

- In a pure hadronic picture a large symmetry repulsion will allow large values for the maximum mass that can be reached by a $N S$, up to more than 2 solar masses $\left(M_{\odot}\right)$. This appears consistent with some recent observations 3 .

- A large symmetry repulsion will favor an earlier transition to a mixed hadron-quark phase at lower baryon densities in the very neutron rich matter of $N S \mathrm{~s}$.

During the discussions the importance of Exchange Terms (the Fock Terms) in the evaluation of spin-isospin contribution to the nuclear EOS has been stressed many times. I have a comment on this point for the young students, a kind of paradox. In all Skyrme effective interactions the isospin dependence is COMPLETELY coming from Exchange Terms. No new isovector parameters are needed!

A common problem for all these repulsive hadronic EOSs in neutron rich matter is the fact that the limit of a direct $\left(p+e^{-} \rightarrow n+\nu_{e}\right) U R C A$ fast neutrino cooling process (proton fraction $Z / A \simeq 0.11$ ) can be reached already at relatively low densities, around $2-3 \rho_{0}$. The point is that we can expect various $N M$ phases at high baryon density in the inner core and this will influence the maximum masses 
as well as the cooling of a NS. Many talks have been devoted to this topic. We must note how the various "paths" of the matter are depending on the scarcely known behavior of the symmetry energy at high density.

In this respect a nice result has been presented by $L . B o$ within a Relativistic Mean Field $(R M F)$ approach. In an hadron+lepton scheme the equilibrium MassRadius relation is largely influenced by the effective fields in the isovector channels, even at the level of a different density dependence of the couplings. The maximum masses appear not much affected, always around $2 M_{\odot}$, but for lower masses we can

have very different corresponding $N S$ radii 4 . This also shows the importance of more data on the mass/radius relation for a large spectrum of $N S$ masses.

The onset of Hyperon Matter has been discussed in several talks (U.Lombardo, L.Dang, T.Maruyama, T.Takatsuka). It will happen when the nucleon chemical potentials will reach the value of the hyperon ones. We remember that hyperons do not have the Fermi kinetic contribution and so such transition is favored. The symmetry energy is also important, e.g. to form lambdas from neutrons the conditon $\mu_{n}=\mu_{\Lambda}$ is better satisfied if the neutrons see a more repulsive symmetry potential. The problem is that when hyperons are formed the matter becomes much softer since we have new degress of freedom and an attractive hyperon interaction 516 . The result is that the expected maximum masses are within two limits, a lower one around $1.3 M_{\odot}$, in order to reach high densities in the inner core, and an upper one below $1.5 M_{\odot}$.

In fact the $Y Y$ interaction is not well known, in particular in the nuclear medium. T.Takatsuka was mentioning the $N A G A R A$ event,, 7 pointing to a smaller attraction in the $\Lambda \Lambda$ system, as a nice indication of some more repulsive terms.

Kaon $\left(K^{-}\right)$condensation is also expected when $\mu_{n}-\mu_{p}=\mu_{e}=\mu_{K}$ (T.Maruyama). Again the symmetry energy is important since we have also $\mu_{n}-\mu_{p}=4 E_{\text {sym }}(\rho)$. Effects on neutrino cooling are predicted via direct URCA processes of baryonic quasiparticles 9 . A fancy $K$ - Pasta structure has been suggested by T.Maruyama due to coulomb effects. Observables?

\section{Quark EOS and Hybrid Stars}

Many talks were addressing the possibility of a transition to a deconfined quark matter in the inner core (U.Lombardo, T.Maruyama, X.Huang, C.Y.Gao,M.Di Toro). Here we directly see the uncertainty due to our poor knowledge of a partonic EOS. The consequences are already at the level of the corresponding $N S$ maximum masses for the relative Hybrid - Stars.

Classes of quark EOS s that privilege the confinement, like the MIT Bag Models, can sustain even masses of $\sim 2 M_{\odot}$ while the $N J L$-like models, that have the correct chiral properties, give instability conditions for masses above $\sim 1.5 M_{\odot}$. The inclusion of pairing correlations in the quark phase are also influencing the equilibrium properties, as nicely shown by C.Y.Gao.

The impression is that we are approaching an important convergence on some 
more fundamental effective field models, like the $N J L$, just introducing new terms that allow the confinement- deconfinement transition. In this way the compact star structures will supply a very interesting arena for testing effective-QCD theories. A good review of the work at the Peking University in the same direction has been presented by Y.X.Liu, including some first results with the Polyakov-Loop implemented $N J L$ model, $P-N J L$. Again we see an effect of the symmetry repulsion, that can lead to an "earlier" (lower density) transition to a mixed hadron-quark phase in the core 10 .

From the point of view of the expected neutron cooling X.Huang has nicely shown how a direct URCA process $\left(u+e \rightarrow d+\nu_{e}\right)$ can develop in the quark phase

In conclusion a picture is emerging of roughly two main classes of neutron stars, one of larger maximum mass (around $2 M_{\odot}$ ) and faster cooling, nucleonic stars or with a repulsive new phase in the inner core, the second with smaller maximum masses (below $1.5 M_{\odot}$ ) and slow cooling, mostly due to modified URCA processes or nucleon-neutrino bremsstrahalung. A corresponding different evolutionary scenario has been recently proposed by P.Haensel 11 .

\section{NS Crust}

Effects of pairing on the low density surface region of a $N S$ or of a very heavy nuclear system have been addressed by J.G.Cao using a RMF approach coupled to a Gogny (non relativistic) treatment of the pairing interaction. The results for the neutron gas distribution are intersting but the use of the $R M F$ parametrizations at such low densities $\left(\rho \sim 10^{-3} \rho_{0}\right)$ has to be justified. We remind that the free couplings at zero densities are not recovered in the model.

T.Maruyama has also presented a very interesting study of the Pasta nuclear structures in the $N S$ crust expected to minimize the total energy in a competition between surface and coulomb effects, again in a $R M F$ nuclear scheme. We see a nice transition from droplet to rod, slab, tube and bubble non uniform structures. I would like to remind that the true Italian Pasta has presently 137 ( the fine structure constant?) different topological structures, better minima could be certainly obtained!

\section{Constraining the Symmetry Energy with Heavy Ion Collisions}

In a meeting on Heavy Ion Collisions and Neutron Stars the Symmetry Energy represents a key issue. In fact the analysis of HIC data is the only tool to extract Symmetry Energy information in region far away from saturation, in particular at the low and high densities of interest for Neutron Stars, as seen before. However the access to the density dependence of the symmetry energy in terrestrial laboratory is limited by three main reasons: $i$ ) the reduced isospin asymmetry of the available interacting nuclear systems that decreases the dynamical effect of the symmetry term; ii) the limits on the density regions that can be probed, up to $2-3 \rho_{0}$; iii)the 
presence of sequential decays from the primary reaction products that can severely wash up the isospin signal.

For all these reasons we need a cross check of many isospin sensitive observables before drawing any firm conclusion. The situation appears rather analogous to the case of the Liquid-Gas Phase Transition in $N M$. We have required more than ten indipendent observables before confirming the effect. Along this line many new ideas have been presented at the conference.

\section{Probing the Symmetry Energy at Low Density}

For the study of the symmetry energy at sub-saturation densities several observables have been suggested via dissipative heavy ion collisions from low to Fermi energies.

\section{Dissipation Dynamics}

At energies just above the Coulomb Barrier the Prompt Dynamical Dipole seems to be rather promising. It represents a fast bremsstrahlung radiation during the charge equilibration dynamics in dissipative collisions initiated in charge asymmetric entrance channels ( $M . D i$ Toro). The symmetry energy acts as a restoring force and the fast neutron emission, even related to the symmetry potentials, is ruling the damping.

Moreover such fast photon emission in charge-asymmetric entrance channels could also be an interesting cooling mechanism in passing from a warm" to a "cold" fusion, with a related larger survival probability of the residue. This could be tested with measurements of fission times (see D.Boilley talk) vs entrance channel asymmetry.

It is also possible to show that the symmetry term is influencing the dissipative dynamics in deep inelastic and fusion reactions with very neutron rich systems. Y.Iwata has presented some evidences of a fusion hindrance due to symmetry effects for the $132 S n+132 S n$ reaction compared to less exotic cases.

\section{Fast Nucleon Emission}

The isospin content of the fast nucleon emission should reveal a good sensitivity to the symmetry potentials. B.Tsang has show some recent data from MSU in reactions $(124,124)$ and $(112,112) \mathrm{Sn}$ on $\mathrm{Sn}$ at $50 \mathrm{AMeV}$. In order to eliminate secondary decay effects a double ratio, n-rich vs n-poor $\mathrm{N} / \mathrm{Z}$ results, is analysed. Some effect is observed in the variation of the isospin signal with the nucleon kinetic energy, but for such studies a mass selection is essential in order to separate different clusters. A similar analysis, but for fragments in multifragmentation reactions, has been proposed by M.Di Toro. We have now an isospin distillation effect, typical of a two component liquid-gas phase transition, coupled to a radial flow. The consequence is a symmetry energy dependent velocity distribution of the produced fragments. 


\section{Isospin Transport}

The isospin transport during the collision should be very sensitive to the symmetry energy at the density of the medium (diffusion term) and to its density gradient (drift term). Therefore several related measurements have been proposed. The direct Isospin Equilibration dynamics at the Fermi energies has been addressed by B.T sang M.Di Toro and L.W.Chen through the study of the "Imbalance Ratio" at the $P L F / T L F$ rapidity. This is an evaluation of the isospin content of the isospin asymmetric collision normalized to the corresponding symmetric cases 12 . In this way the secondary decay effects are expected to be much reduced. The centrality dependence of such quantity appears sensitive to the stiffness of the symmetry energy, of course self-consistent transport calculations are needed. A way to direct measure this effect could be to analyse the correlation to the amount of kinetic energy dissipation.

L.W.Chen has focussed the attention on the uncertainties coming from the momentum dependence of the effective interactions, that are reducing the interaction times, and from the in medium corrections to the nucleon nucleon cross sections. Anyway some first conclusions seem to converge on a rather stiff behavior of the symmetry term around saturation, with a density dependence $E_{\text {sym }} \approx\left(\rho / \rho_{0}\right)^{\gamma}$ with $0.7<\gamma<1.1$. Since the asymmetric matter compressibility is also affceted by such

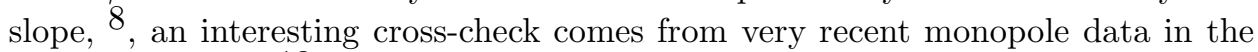
Sn isotopic chain 13 .

The neutron enrichement of mid-rapidity fragments for ternary events (neck fragmentation) seems to confirm this relatively large gradient of the symmetry term around saturation. This point is not trivial since a large class of effective interactions can be dropped and more diffuse neutron skin distributions in neutron excess systems can be expected.

\section{Neutron Skin}

Unfortunately the neutron skin thickness cannot be directly tested by elementary probes. Z.X.Li has suggested a new observable, the proton-nucleus reaction cross section at intermediate energies, that should be rather sensitive to the neutron distribution. The argument is based on Isospin-QMD transport simulations, but the physics behind appears very clear. As already noted a stiff symmetry energy implies a larger n-thickness but together with a less attractive proton potential. The result will be a smaller reaction cross section for proton nucleus collisions when the $\mathrm{N} / \mathrm{Z}$ of the target is increasing. Systematic measurements for isotopic chains are needed.

D.Q.Fang is proposing the use of the Statistical Abrasion Ablation model, limited to PLF neutron abrasion events, to reconstruct the neutron skin structure of the target. 


\section{Collective Radial Flow}

F.S.Zhang has tried to see a symmetry energy dependence of the threshold of collective flows, in particular the radial flow, in collisions at the Fermi energies. The threshold is related to the onset of compression-expansion dynamics, which is ruled by the stopping power of the NN cross sections and the nuclear matter compressibility. The latter is depending on the symmetry energy (slope and curvature) but for the asymmetries tested with stable isotopes the effect appears neglgible (of the order of $1 \%$ for a large variation of the symmetry stiffness). Much more important is the dependence on the used in-medium cross sections.

\section{Isoscaling}

The use of the Isoscaling signal in order to extract the symmetry energy at low densities has been presented by W.D.Tian, M.Veselsky and W.Trautmann. It consists in the relation $R_{21}=Y_{2}(N . Z) / Y_{1}(N, Z)=C \exp (\alpha N+\beta Z)$ where $Y_{i}(N, Z)$ are the yields for the $(N, Z)$ fragment measured in two different reactions, n-rich vs n-poor.

The connection to the symmetry energy is clear in an equilibrium gran canonical model of the multifragmentation. The relation is not so evident in a dynamical approach where the isoscaling parameters appear also depending on the widths of the primary isotopic and isotonic distributions 14 . The fact that the isoscaling signal has been observed in a very different fragment production mechanisms, from deep-inelastic to fission, as well as in events where fragments are showing clear dynamical features (like neck fragmentation) seems to suggest some caution in the direct use of statistical approaches.

Some new projectile fragmentation data have been shown by W.Trautmann where the isospin asymmetry is enhanced by the use of unstable beams (107Sn and $124 \mathrm{La}$ ) vs the n-rich reference system $124 \mathrm{Sn}$. A clear centrality dependence of the isoscaling parameters is observed: the $\alpha$ is decreasing for more central events indicating less n-rich fragments. This can be due to a neutron distillation during the primary fragment formation as well as to larger neutron sequential emission. Neutron detection as well as a reconstruction of the primary fragments seem to be very important.

Finally the effect of isospin momentum dependent forces on the neutron distillation has been discussed by $J . X u$. We see a slight increase of the mixed phase region where the distillation takes place. The poorly known temperature dependence of the symmetry energy could explain this result.

\section{Probing the Symmetry Energy at Supra-Saturation}

The way to test the behavior of the Symmetry Term at high densities, of large importance for Neutron Star Modeling, has been addressed by L.W.Chen and M.Di Toro. Here we need beam energies at least of few hundred AMeV in order 
to reach regions of 2-3 times $\rho_{0}$ during the compresssion stage. Therefore transport simulations should have at least a relativistic kinematics. In fact we can see that even a fully covariant treatment of the interactions is very relevant $8115[16$.

There is a general agreement on the observables more sensitive to the stiffness of the symmetry energy in this density range, but very few data are presently available. A general comment is that we need high transverse momentum selections when we try to see reaction products coming from the transient high density stage. This largely reduces the multiplicities... and the happiness of the experimentalists.

Good obervables appear to be neutron/proton (or triton/3He) ratios and flow differences. For the latter we expect even an important fully relativistic effect due to the Lorentz forces, present in the relativistic dynamics, that enhance the contribution of the vector-isovector part of the interaction 15 .

Rather promising seems to be the study of yield ratios of mesons with different isospin structure, like $\pi^{-} / \pi^{+}$and $K^{0} / K^{+}$. In fact the isospin effects on the inelastic vertices are directly related to the nucleon/meson self-energies and so the corresponding rates will be very sensitive to the isovector covariant structure of the symmetry term. This is particularly true around the production thresholds $\frac{16}{\text {. But }}$ we cannot ask a $p_{t}$ selection over there......

Certainly highly isospin asymmetric unstable beams in this energy range will largely improve the overall experimental sensitivity.

Increasing the beam energy we can reach higher baryon densities but many new inelastic channels are opening, with increasing uncertainties on the relative cross sections. An interesting contribution in the direction of the possibility of observing new phenomena has been delivered by B.S.Zuo. Focussing on data from $p p$ collisions he has shown the importance for the strangeness production of the components beyond the 3-quark structure of the nucleon resonances, in particular for $N^{*}(1535)$ and $\Delta^{*}(1620)$. It was an hadron structure talk but we can easily see the implications for heavy ion collisions at intermediate energies.

\section{Fragmentation Simulations for Cancer Therapy}

At the end of this section mainly devoted to fragmentation results I would like to mention the contribution of B.A.Bian with some results of fragment production simulations for $12 \mathrm{C}$ and $20 \mathrm{Ne}$ beams at Fermi and Intermediate enegies on targets of interest for HIC cancer therapy. This kind of studies are extremely important and the topic should require much more attention in the near future, even considering the improvements of the transport approaches. We need also more data to compare with. Moreover the angular distributions of the reaction products will be very important. E.g. it would be nice to check if prompt break-up events at forward angles can be correctly accounted for within the mean field plus fluctuation model discussed here. 


\section{Particle correlations and Collective Flows}

\section{Imaging}

As already noted before, the reconstruction of primary sources of the final reaction products is of fundamental importance. G.Verde has shown the high degree of accuracy reached with the measurements of particle-particle correlation. Of course the "Imaging" technique is always based on the inversion of an integral equation and so experimental uncertainties are always present. Some very nice new results have been presented: i) on effects of the symmetry energy stiffness on pp correlations (in a soft symmetry term the protons are more attracted); ii) on a direct study of emission hierarchy, (different particles and clusters probe different sources, as expected by dynamical models); iii) on the competition with temperature effects.

The interest even for spectroscopic studies has been shown from the analysis of $12 \mathrm{C}$ projectile fragmentation data. $\alpha$-correlations in excited states point to resonance structures, in particular to the famous Hoyle Resonance, of fundamental importance for the synthesis of the elements and the formation of organic matter.

The short emitting times detected from pion interferometry in $\mathrm{Au}+\mathrm{Au}$ collisions at the ultrarelativistic RHIC energies have inspired the Granular QGP model presented by $W, N$.Zhang. The saturation of the elliptic flows at high transverse momentum is also accounted for in the same picture, although a decrease for larger $p_{t}$ is predicted, not observed so far. The cluster structure of the expanding QGP is suggested even in hadronization models via a spinodal mechanism in a first order phase transition scheme (see the M.Velsesky report).

\section{Scaling of Collective Flows}

A thorough analysis of anisotropic collective flows has been delivered by Y.G.Ma, ranging from Fermi to ultrarelativistic energies. The stimulating suggestion is that similar scaling properties should be observed in the two very different energy regimes just comparing hadron flows vs. quark composition with respect to light ion flows vs. nucleon composition. The similarity is based on a common coalescence mechanism for the hadron/ion formation. The idea is very suggestive.

In fact at RHIC energies such scaling has been observed for hadron flows in $\mathrm{Au}+\mathrm{Au}$ collisions at $200 \mathrm{GeV} / \mathrm{c}$. At the Fermi energies no evidence is presently available. Y.G.Ma suggests to revisite the available flow data accumulated in the last 20 years. His point is based on light ion $(A \leq 4)$ results derived from $B U U$ transport simulations for $86 \mathrm{Kr}+124 \mathrm{Sn}$ collisions at $25 \mathrm{AMeV}$, stopped at $200 \mathrm{fm} / \mathrm{c}$, very early for this energy region. Unfortunately the low energy heavy ion dynamics is much more complicated and this interesting signal can be easily washed out by later cluster emissions of different nature, dynamical as well as statistical. In any case the idea is stimulating, maybe for cluster formation at higher energies, where the final state interactions can be less important. A second message of this interesting talk is too look at the hard gamma flows, always in the Fermi energy 
domain. An anticoincidence with neutron/proton flows is expected for the high energy part of the gamma spectrum, of non thermal origin.

\section{Looking for SHE}

I feel a profound admiration for the very brave people trying to access a fusion dynamics at the level of the pico-barn cross sections. We have seen the problems and the status of the art in the nice talks of Y.Abe, E.G.Zhao and D.Boilley. All the approaches are based on a Brown-Motion inspired treatment, with large dissipationfluctuation effects. In this respect it has been rather impressive the picture of the fusion-fission trajectories in the deformation-separation plane shown by Abe-san. Since we are facing a two-steps transport problem, coulomb and shape barriers, it is clear that the fluctuation dynamics can give a substantial contribution. The main problem is the choice of the relevant degrees of freedom.

We need reactions with large neutron excess to better enter the expected stability island ( $\mathrm{Z}$ between 114 and 126, N between 172 and 184, depending of models). In this respect the use of unstable neutron rich elements could be helpful, but the effect of neutron abundance on the residue cross section is not yet clear, see the E.G.Zhao report.

In my opinion a very good step forward in the direction of a better testing of various fusion-fission models is related to the possibility of measuring long fission times using the Blocking Technique in Crystals, as shown by D.Boilley 17 . The fact that it has been possible to discriminate between $238 \mathrm{U}+\mathrm{Ni}(\mathrm{Z}=120$ compound) and $238 \mathrm{U}+\mathrm{Ge}(\mathrm{Z}=124$ compound) reactiions, with evidence of long fission time events $\left(\tau>10^{-18} s\right)$, at variance with the $208 \mathrm{~Pb}+\mathrm{Ge}(\mathrm{Z}=114$ comp. $)$ case, represents a very promising result. Of course there are limitations for the target choice, in particular the $\mathrm{N} / \mathrm{Z}$ selection.

\section{Short Notes}

I have appreciated same "special" Characters during the Conference:

- Y.M.Zhao: The Highly Random

He suggests a nuclear spectroscopy based on Random Interactions

- T.Maruyama: The Highly Mixed

He sees Mixed Phases everywhere, with very exotic shapes

- G.Verde: The Highly Correlated

He can disentangle correlation effects in any possible system

- Y.G.Ma: The Highly Scaled

Scaling is always there, in any response of a complex system

\section{Conclusions}

I hope that I have been able to express my deep feeling that we have enjoyed a very lively and stimulating meeting, in spite of the fact that certainly I have missed 
several important points raised during the presentations and the discussions. The very friendly atmosphere of the Beijing Normal University and the warm support of the Physics Department Group, lead by Prof.F.S.Zhang, have largely contributed to the success of the Conference.

Finally I have still to remark the very nice contributions of several young people from many different Chinese Universities and Research Centers. This is the best way to show the excellent development of the field in China. In this respect I think important to finish with the very best wishes for the new Lanzhou Cooling-

Storage-Ring facility. We have heard from Prof. J.S.Wang that everything is going on very successfully: before the end of June 2007 three beams have been extracted, stored, cooled and accelerated up to $1 \mathrm{AGeV}, 12 \mathrm{C}\left(210^{9} \mathrm{pps}\right), 36 \mathrm{Ar}\left(1.410^{9} \mathrm{pps}\right)$ and $129 \mathrm{Xe}\left(10^{8} \mathrm{pps}\right)$. Congratulations and looking forward to a very exciting physics!

\section{References}

1. X.R.Zhuo, G.F.Burgio, U.Lombardo, W.Zuo Phys.Rev. C69 (2004) 018801.

2. P.Danielewicz et al., Science 298 (2002) 1597.

3. D.Page, S.Reddy, Ann.Rev.Nucl.Part.Sci. 56 (2006) 327.

4. B.Liu et al., Phys.Rev. C75 (2007) 048801.

5. G.F.Burgio et al., Phys.Rev. C61 (2000) 055801.

6. I.Vidana et al., Phys.Rev. C73 (2006) 058801.

7. H.Takahashi et al., Phys.Rev.Lett. 87 (2001) 212502.

8. V.Baran, M.Colonna, V.Greco, M.Di Toro, Phys.Rep. 410 (2005) 335.

9. D.G.Yakovlev et al., Theory of cooling neutron stars versus observations arXiv:0710.2047 [astro-ph].

10. M.Di Toro et al., Nucl.Phys. A775 (2006) 102.

11. P.Haensel, Contribution to the EXOCT Conference, Catania June 2007, World Sci. in press.

12. F.Rami et al., Phys.Rev.Lett. 84 (2000) 1120.

13. U.Garg et al., Notre Dame - Osaka Exp., arXiv, nucl-ex/0709.3132

14. T.X.Liu et al., Phys.Rev. C69 (2004) 014603.

15. V.Greco et al., Phys.Lett. B562 (2003) 215.

16. G.Ferini, T.Gaitanos, M. Colonna, M.Di Toro, H.H.Wolter. Phys.Rev.Lett. 97 (2006) 202301.

17. M.Morjean et al., Eur.Phys.Jour. D (2007) in press. 